\title{
DEVELOPMENT AND TESTING OF A SMALL SCALE WAVE ENERGY CONVERTER AND FULLY CONTROLLED LINEAR ELECTRIC GENERATOR
}

\author{
N.J. Baker ${ }^{*}$, L. McNabb ${ }^{2}, S$ McDonald ${ }^{1}$, A. Almoraya ${ }^{1}$ \\ ${ }^{I}$ School of Engineering, Newcastle University, Merz Court, NE1 7RU, UK' \\ ${ }^{2}$ School of Engineering, RMIT University, GPO Box 2476, Melbourne VIC 3001 Australia \\ *nick.baker@newcastle.ac.uk
}

\begin{abstract}
Keywords: WAVE ENERGY CONVERTERS, LINEAR GENERATORS, VOLTAGE SOURCE CONVERTERS, MAXIMUM POWER POINT TRACKING, HILL CLIMB SEARCH
\end{abstract}

\begin{abstract}
This paper presents the development of an electrical drive suitable for energy conversion in an ocean wave energy converter. Two representative wave energy converters are used to design a linear generator and associated power electronic converter. The electrical drive is built and coupled to two small scale hydrodynamic prototypes which are tested in a wave tank. The operation of the drive, including variation of apparent damping and spring coefficients is presented and used to demonstrate automated direct drive control via a maximum power point tracking algorithm.
\end{abstract}

\section{Introduction}

The UK's target to have "net zero greenhouse gas emissions by 2050" implies that interest in renewable electricity generation is going to continue for the foreseeable future. One option is to capture the energy contained in the surface waves of the ocean. Over the past few decades many 'direct-drive' concepts of extracting wave energy have been proposed at small scale, such as [1-3], and a handful have been demonstrated at full scale e.g. [4-8]. However, there is no technological consensus as to what a commercially successful Wave Energy Converter (WEC) will look like. This is a challenge for drive designers wishing to propose generic power take off units in this sector.

The majority of WEC devices have some form of oscillating prime mover from which electrical power can be extracted. The period of oscillation for high energy sites is around 10 seconds, and if a peak to peak amplitude of $4 \mathrm{~m}$ is assumed, this gives a peak WEC velocity of $1.2 \mathrm{~m} / \mathrm{s}$. Such a low peak velocity is a challenge for the power take off, especially when compared to the air-gap speed of a typical electrical machine. As power is the product of force and velocity, any reciprocating motion necessarily has periods of zero power. In addition, if there is a desire to actively control the oscillation of the wave energy device, for example to maximise power capture, then a fully rated power converter with energy storage and bi-directional power capability is required.
This paper describes the design and demonstration of an allelectric drive train capable of extracting power from a WEC, including exporting electrical power and implementing oscillation control.

\section{Type of wave energy converter}

For those interested in designing an electrical drive train for this application, research either has to align with a specific WEC developer, or various assumptions need to be made to produce technology of interest to a wide variety of device concepts. In this work, two generic WEC's have been used to design and demonstrate the method of wave energy power capture and the associated power take off control. 


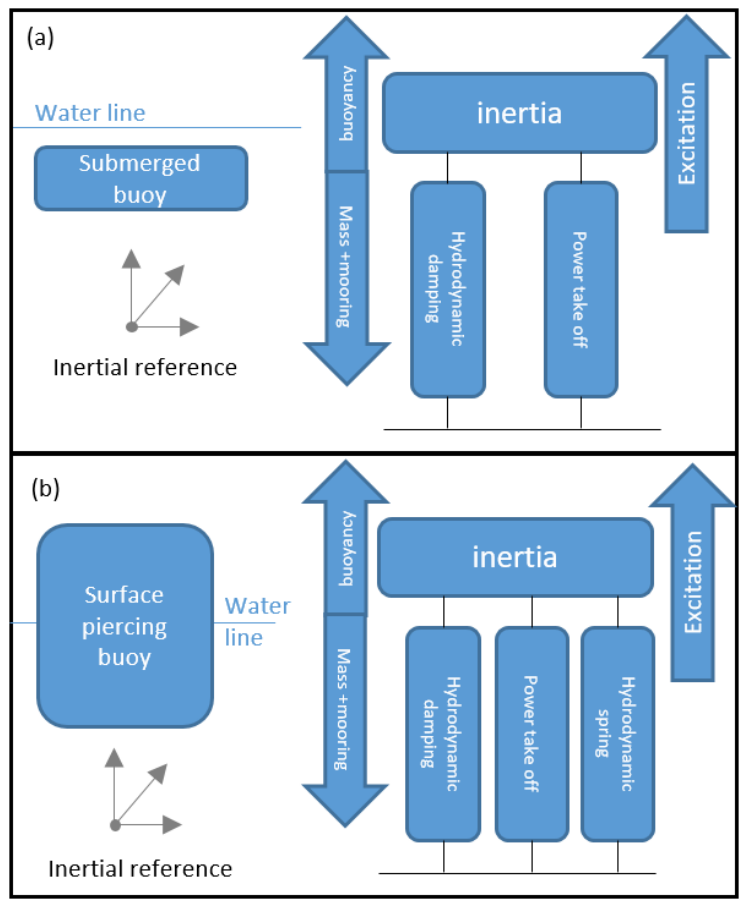

Fig. 1 Representative heaving WEC's. (a) A fully submerged buoy, (b) A surface piercing buoy.

Fig. 1 (a) shows the schematic of a fully submerged buoyant vessel and its simplified force representation. In calm water, the buoyancy forces are in equilibrium with the mass and any external mooring forces. Any oscillation is resisted by a hydrodynamic damping force, and energy is extracted by further opposing the motion with a power take off. Surface waves exert an excitation force on the body, either due to a change in surrounding water pressure or due to particle motion depending on the depth. The amount of power extracted can be altered by controlling the power take off force. In many systems, it is assumed this is proportional to velocity and so the power take off acts as an additional damping force, and control is achieved by varying the effective damping coefficient. This buoy is representative of Carnegie's CETO device [8] and other wave energy devices.

Fig. 1 (b) shows the equivalent diagram for a surface piercing buoy. Here, variation of the buoy's position relative to the water line varies the buoyancy force. In the force representation, this appears as a hydrodynamic spring force. This system now has a natural frequency, and in many devices it is generally accepted that the most effective way of extracting power is to operate the device near its resonant frequency. If the power take off is fully controllable, as is the case for a converter connected electrical machine, the resonant frequency of the device can be altered by altering the effective spring constant of the power take off as well as the damping factor. i.e. making a component of the generator force a function of buoy position and is termed mechanical reactive power control [9]. This system behaviour is representative of many heaving buoy type wave energy converters e.g. [4, 6-8]. In this paper the two parameters used to specify the desired power take off force at any given time are defined as $K_{v e l}$ for the velocity dependent damping and $K_{p o s}$ for the position dependent spring force.
Demonstrating the effect of varying $K_{v e l}$ and $K_{p o s}$ is representative of demonstrating power take off control in a WEC and in this paper is presented at small scale for both the buoys considered, i.e. concepts with and without a spring force. Successful implementation implies that this approach can be applied at full scale for a wide range of WEC designs and concepts.

\section{Linear Generator}

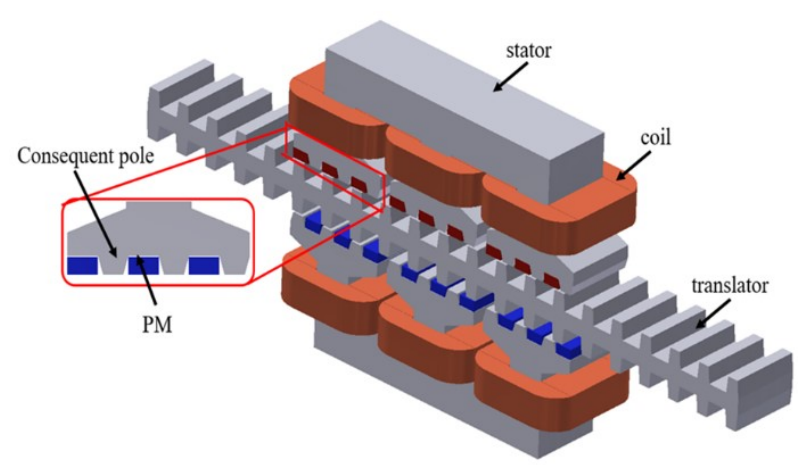

Fig. 2. Mode of the Inset Magnet Consequent Pole Vernier Hybrid Machine

In either of the buoys of Fig. 1, for power take off systems with just one degree of freedom, e.g. through a mooring line, the majority of useful power is derived from the buoy's oscillating motion with a relatively low speed and high force. This motion is not well suited to utilizing a rotary electrical machine without some form of gearing. A linear generator such as the Vernier Hybrid Machine (VHM) is potentially well suited to this motion. The high shear stress capability of the VHM makes it attractive as a generator in cases where a conventional linear or rotating machine would be physically very large. It is a flux switching Permanent Magnet (PM) machine where the magnets and coils are mounted on the stator, leaving the translator to be a purely iron structure. A known limitation of this machine class is the relatively poor power factor, which adversely affects the power converter rating and hence system cost. 


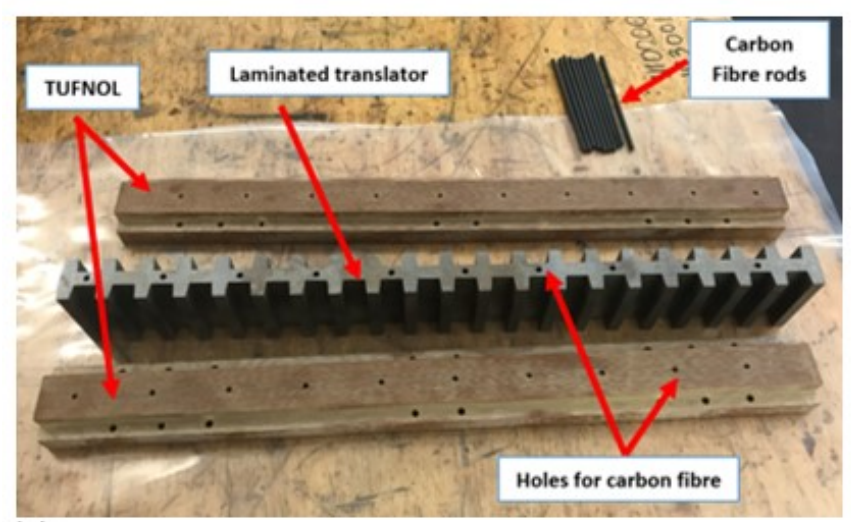

(a)

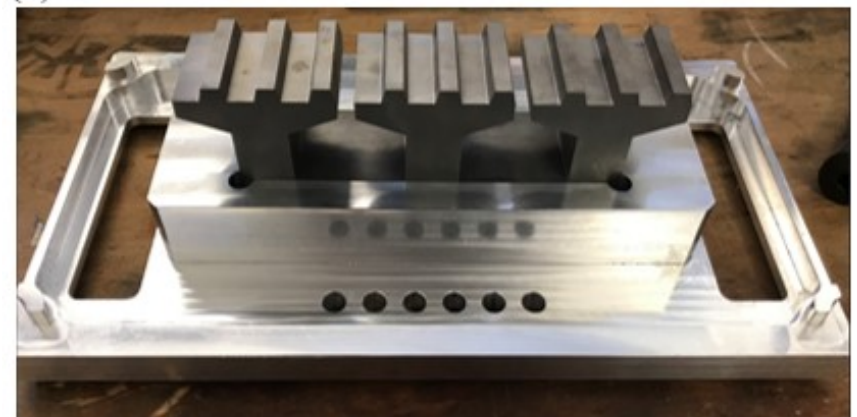

(b)

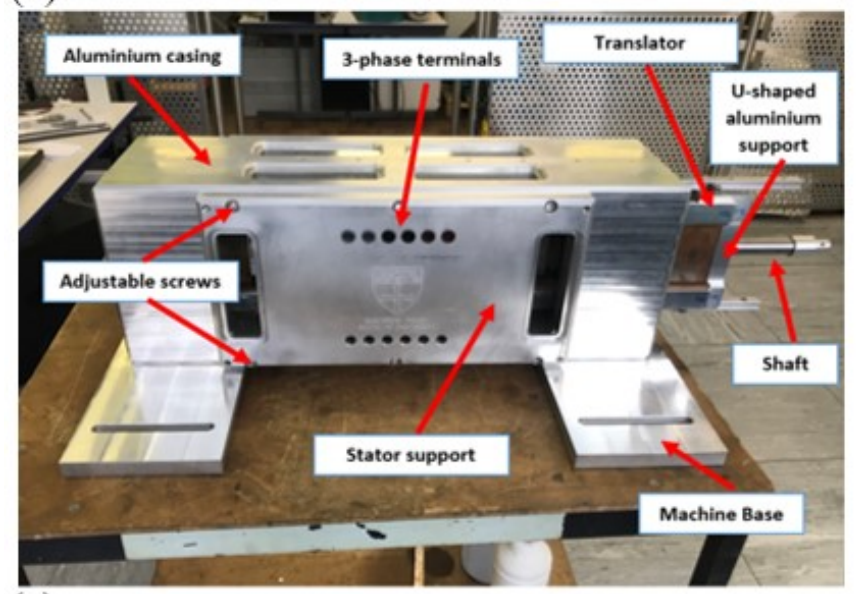

(c)

Fig. 3. Build of the linear machine (a) translator laminations (b) stator laminations and (c) fully assembled in the housing.

A consequent pole structure can be employed in this family of PM machines for the purpose of minimising the magnet consumption [10] and improving improved torque/ force density [10-13]. For wave energy applications, other Vernier machines with a consequent pole structure have been proposed in [14].

The machine topology used in this paper comprises of two balanced stators with identical E-cores facing each other upon which both the PMs and windings are located, as shown in Fig. 2. The long iron laminated translator with salient teeth is sandwiched between the stator sides prior to being mounted in an aluminium housing as shown in Fig. 3.

\section{Converter}

The power train of a wave energy device is essentially a variable-speed-drive, consisting of an electrical machine, an AC/DC machine side converter, a DC bus (potentially including energy storage) and a DC/AC grid side converter. The generator charges the DC-link capacitor via a Voltage Source Converter (VSC) and then a Voltage Source Inverter (VSI) is used to convert the DC-link voltage and current into grid frequency $\mathrm{AC}$ voltages and currents. For a constant speed application this arrangement works well and the various components can be optimally selected. However, in wave energy there are a number of additional requirements which influence the drive design. For example the generator is typically accelerating from zero to full speed and back to zero every few seconds. There is also an increased emphasis on reliability and fault-tolerance, as servicing offshore platforms is weather dependent and loss of the drive means a loss of revenue. Furthermore, if it is required to control and tune the wave energy device to maximise power output, four-quadrant operation is required. Finally, local energy storage to smooth fluctuating real power and also implement the active control associated with tuning the wave energy converter requires significant reciprocating power flows over and above the average power being fed into the grid [9].

To address these requirements, a modular approach is proposed for the power-take-off, whereby multiple electrical generators are mechanically coupled and connected to multiple power converter modules. A bespoke voltage source converter has been built in this project and used to control the linear machine [15].

\section{Hardware Testing}

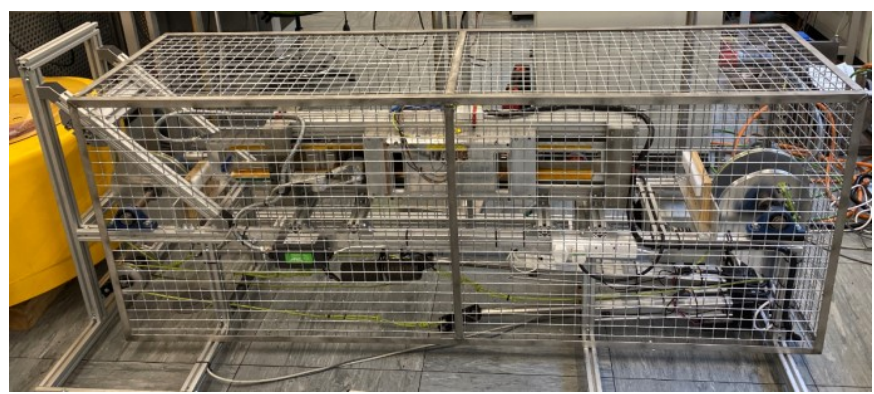

Fig. 4. Linear machine mounted in the testing frame.

To demonstrate the power take off and control concept, a portable test rig was fabricated to house the linear machine of Fig. 3 . The rig has the capability of applying a fixed force to one side of the translator, in order to offset the residual buoyancy force shown in Fig. 1 without injecting current into the generator. This offset force is provided by a mechanical spring, via a cam shape to remove the natural linear variation of force with position inherent within a spring. The force is transferred to a mechanical load via a series of pulleys and rope and the general arrangement is shown in Fig. 4. The rig hence allows the electrical machine to be mechanically coupled to a scale WEC in a test tank via a cable, without being submerged in water as shown schematically and photographically in Fig. 5. The steady-state buoy position can hence be altered without 
a residual force requirement from the machine. The drive is therefore solely concerned with the bouy's oscillation.

Prototypes of the submerged and and semi-submerged bouys shown in Fig. 6 were used to excite the linear drive. Fig. 7(a) shows the electrical power captured from the submerged buoy in a fixed sea state whilst the controller equivalent damping is altered. Fig. 7(b) has the equivalent diagram for the surface piercing buoy, where both damping and spring force is varied.
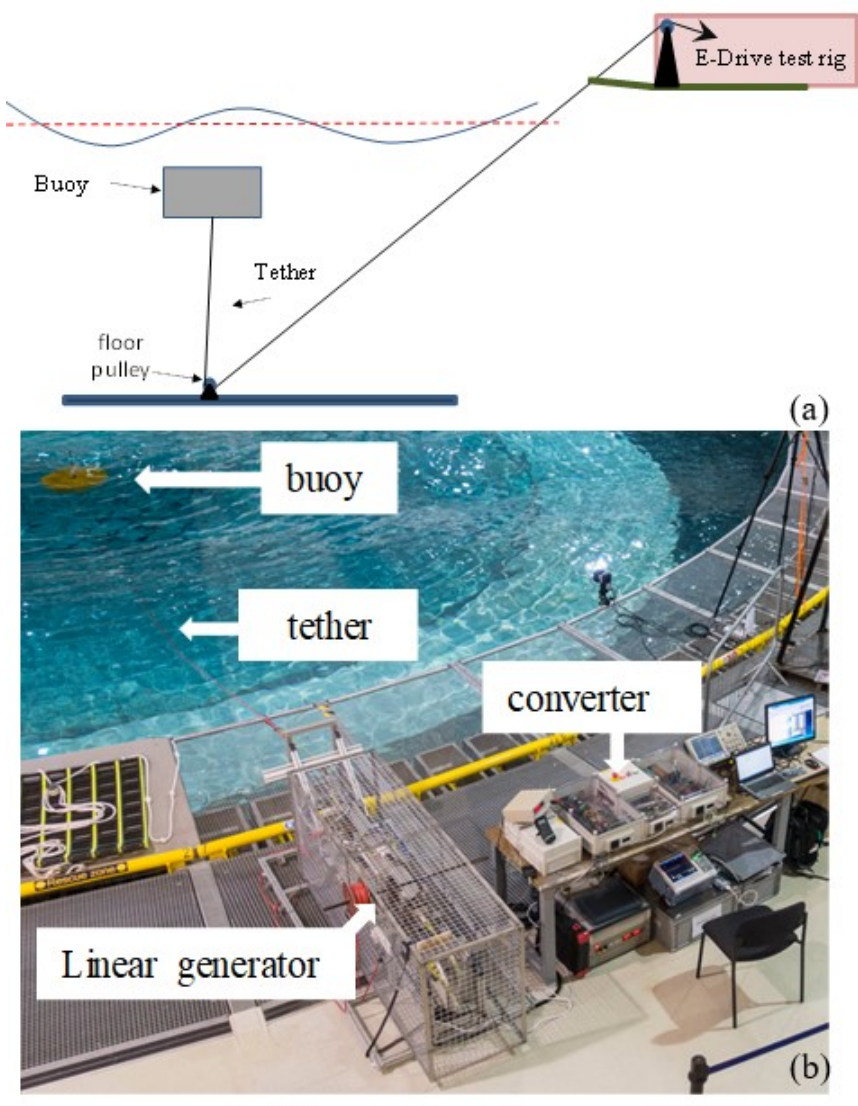

Fig. 5. Test layout (a) schematic and (b) in the wave tank

(a)

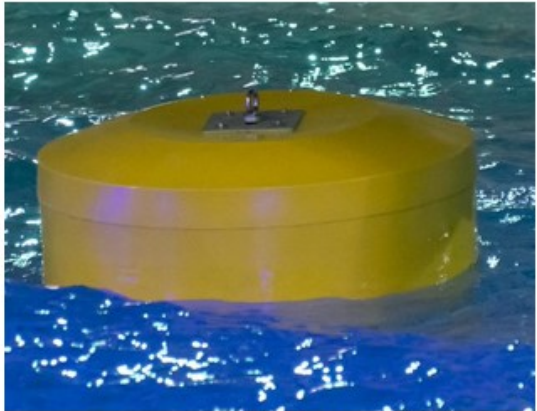

(b)

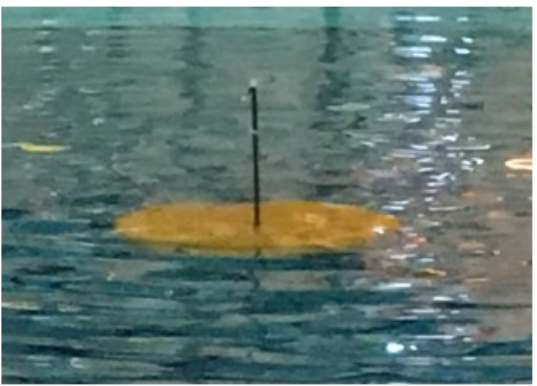

Fig. 6. Buoys used for testing (a) surface piercing and (b) fully submerged

\section{(a)}

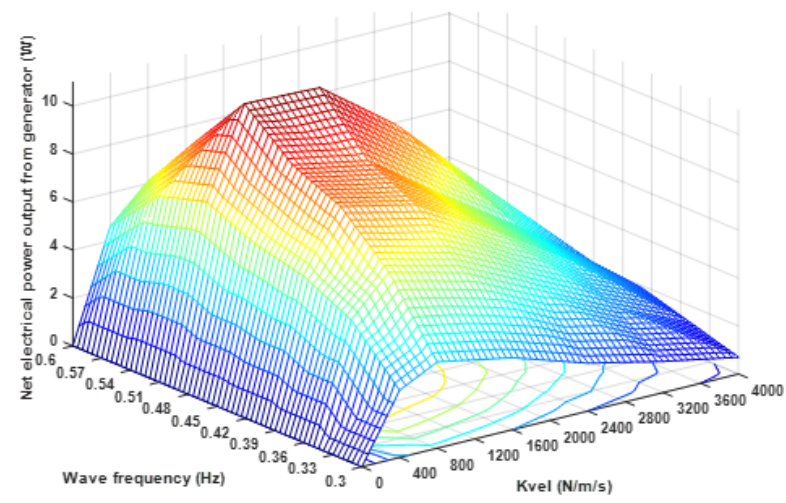

(b)

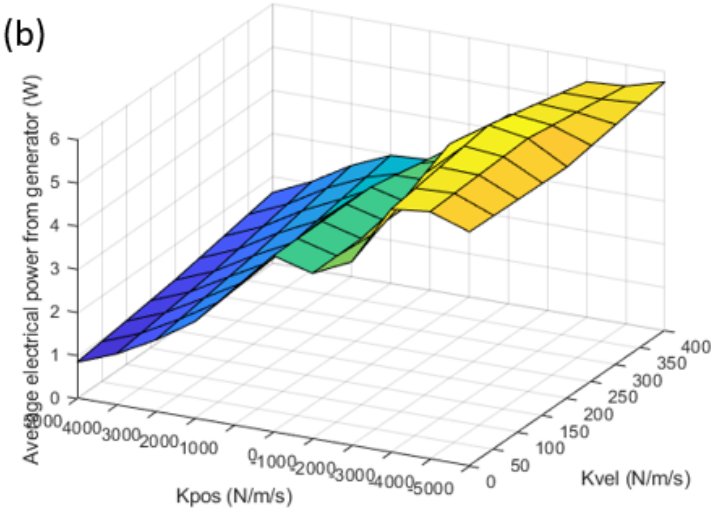

Fig. 7. Power extraction experimental tests showing electrical power output verses damping coefficient $\left(K_{v e l}\right)$ (a) for a range of wave frequencies for the submerged buoy, (b) for a range of spring constant $\left(K_{\text {pos }}\right)$ for a fixed wave frequency tests for the surface piercing buoy.

In both cases, it is shown that variation in the equivalent damping of the generator results in a variation in net electrical power. The maxima observed is analogous to the maximum power transfer occurring in a generator when the internal and external impedance are matched. There is a slight shift in the $K_{\text {vel }}$ required for maximum power with a variation in wave frequency. This is analgous to the effect of electrical frequency on impedance. Fig. 7(a) hence demonstrates that the damping force of a generator can be used to maximise the output power of a wave energy converter which has no residual hydrodynamic spring and hence no natural frequency.

The plot of Fig. 7 (b) refers to a surface piercing bouy being excited at $0.6 \mathrm{~Hz}$, which is close to its natural frequency. In this plot, generator force is controlled to be a function of both position and velocity - i.e. a spring and damping force. Results at low damping can be seen to have two peaks: corresponding to two modes of oscillation in the tank. It demonstrates that variation in the effective spring force of the drive can be used to maximise the output power and that local minima exist in the operation space. 
Efficiency of the system is calculated as electrical power out of the machine divided by mechanical power in. A load cell and the translator velocity measure the mechanical power. The load cell is mounted to the base of the buoy, and so results include the generator force plus friction in the cable, friction in the rig and the mechanical spring force. Fig. 8 shows the equivalent efficiency plot for the power results shown in Fig. 7 (b). It shows that the semi submerged buoy operates at greates efficiency with no spring force $\left(K_{p o s}=0\right)$. There are two things to be inferred here: firstly that control of the spring force adversely affects machine losses, due to increased active current. Secondly, in wave energy devices system efficiency is not a good representation of overall device performance as a seemingly low efficiency operating point can still generate the most power within a given range.

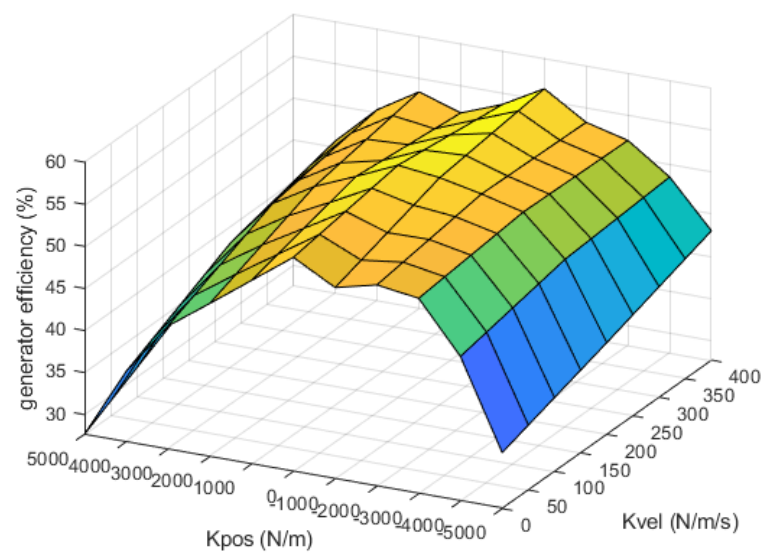

Fig. 8. Generation efficiency for a fixed frequency sea wave for the surface piercing bouy. Variation of damping and spring contants corresponds to that in Fig. 7 (b).

\section{Control algorithm testing}

In real oceanic deployment, the amplitude and frequency combinations of the sea are continuously varying. As such, in order to maximise the power extraction in real time, the damping and spring parameters must be continually adapted to match the WEC characteristics to the given sea.

To demonstrate the principle of automated direct drive control, a 'cycling' maximum power point tracking algorithm (MPPT) $[16,17]$ has be designed and implemented in the test platform. Beyond just demonstration purposes, MPPT algorithms provide a functionally robust and pragmatic solution to the optimisation problem. As a non-model based algorithm, they can be rapidly deployed and quickly tuned online, avoiding many of the difficulties associated with model based approaches such as accounting for complicated multidimensional dynamics [18]. Under simple conditions, such as damping control which yields a convex mapping between the damping factor and output power (see Fig. 7(a)), MPPT based approaches are particularly well suited, as the gradient ascent type algorithm is able to easily assert optimal points $[16,18$, 19]. However, under more complex conditions, such as springdamping control, the performance may be compromised due to the presence of multiple local optimal points associated with the various resonances in each mode of oscillation (e.g. see Fig. 7(b)). As with any locally minimising algorithm, the found solution depends on the initialisation.

The 'cycling' MPPT algorithm that was used is an application of a recent technique developed in [16]. This algorithm in particular improves on the deficiencies of standard 'perturb and observe' type MPPT algorithms in irregular seas. In brief, the alogorthm operates by constantly perturbing the control variable (e.g. damping factor) over a number of sea wave cycles using a sinusoidal waveform. Over a prescribed interval (e.g. taking a few cycles of the perturbation waveform), the statistical covariance between this waveform and the output power is established. Utilising the resulting covariance, the control variable can hence be updated proportionally to the 'strength' of the relationship. The algorithm can be tuned by varying the magnitude of the perturbation waveform to increase the sensitivy/effective region, and, passing the covariance result through an additional gain parameter to modify its effect on the control variable; these can be tuned online with relative ease. A useful trait of the algorithm is that parallel instances using different perturbation waveform periods can be run to control multiple variables. Further details and examples of the cycling MPPT algorithm can be found in $[16,17]$.

In practice, the cycling MPPT algorithm was able to effectively optimise the test platform under damping control action. Fig. 9 illustrates the experimental results of the algorithm under a $0.75 \mathrm{~Hz}$ regular sea with a $0.25 \mathrm{~m}$ waveheight run for seven minutes. MPPT update intervals were one minute. The results illustrate the algorithm actively adjusts the damping coefficient to optimise the output power response, increasing the average output by approximately $30 \%$, and nearly doubling the peak power, as shown. The effect of the control solution is particularly evident in the first 200 seconds of run-time (extending past the initial start-up transient interval which finishes at approximately 30 seconds) where the average and peak power outputs drastically improve.

Further experiemtal studies conducted under the same regular sea conditions with combined spring-damping control were able to demonstrate a small improvement of around $2.5 \%$. On review however, we believe that this result could be readily improved. As discussed, the MPPT algorithm is a locally minimising optimisation scheme which is sensitive to its initialisation (i.e. the initialisation determines which solution the algorithm will be locally convergent to). In the conducted cases, the spring term was initialised at zero, hence, it found local solutions that were damping dominant, and, used only a minor degree of spring action. Characteristially, the geometry of the given bouy tended to exhibit local optimal points at low spring values as illustrated previously in Fig. 7b. Further investigation of this issue is warranted, however, at least in principle reactive power control is demonstrated. 


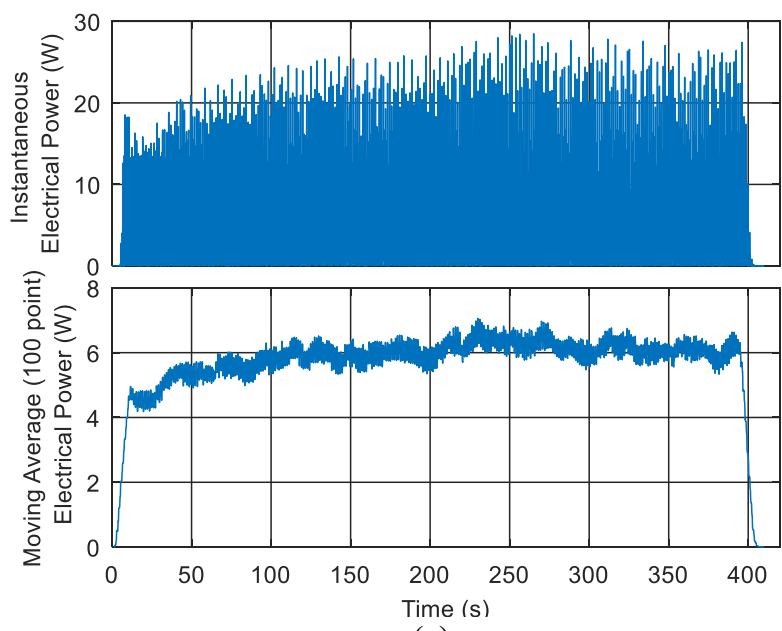

(a)

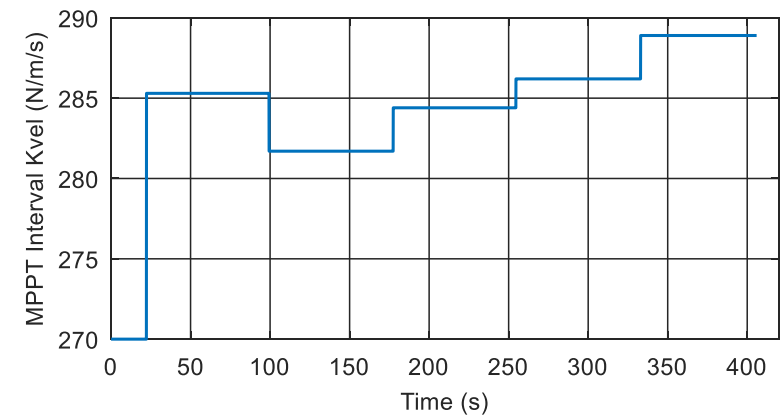

(b)

Fig 9. 'Cycling' MPPT with damping control. $0.75 \mathrm{~Hz}, 0.25$ $\mathrm{m}$ peak-to-peak regular sea run for seven minutes. (a) Instantaneous electrical power \& 100 point moving average electrical power, (b) damping coefficient updated approximately every minute.

\section{Conclusion}

This paper has provided an overview of the development of a linear power take off drive for a direct drive wave energy converter. Via small scale tank testing, it has demonstrated that direct electrical power take off can be used to control the electrical power that can be extracted from two generic forms of wave energy converter by manipulating either the spring or damping term. It has also been demonstrated that those terms can be controlled by an automated maximum power point tracking typw algorithm, which implies that the devices can track the ideal operating coefficients ina varying sea state.

\section{Acknowledgements}

This work has been funded by EPSRC Ref. EP/N021452/1, (EDRIVE-MEC) under SUPERGEN Marine. L McNabb was supported by the Australian Research Council Discovery Project DP170101039. The two bouys and testrig was deigned, manufactured and installed by fountain design Ltd.

\section{References}

[1] X. Yin, X. Li, and L. Zuo, "Dynamic Characteristics and Test Results of a Wave Power Take-off System with Mechanical Motion Rectification and Transmission," IEEE Transactions on Industrial Electronics, 2019.

[2] R. Crozier, H. Bailey, M. Mueller, E. Spooner, and P. McKeever, "Analysis, design and testing of a novel direct-drive wave energy converter system," IET Renewable Power Generation, vol. 7, no. 5, pp. 565-573, 2013.

[3] J. Prudell, M. Stoddard, E. Amon, T. K. Brekken, and A. Von Jouanne, "A permanent-magnet tubular linear generator for ocean wave energy conversion," IEEE Transactions on Industry Applications, vol. 46, no. 6, pp. 2392-2400, 2010.

E. Lejerskog, E. Strömstedt, A. Savin, C. Boström, and M. Leijon, "Study of the operation characteristics of a point absorbing direct driven permanent magnet linear generator deployed in the Baltic Sea," IET Renewable Power Generation, vol. 10, no. 8, pp. 1204-1210, 2016.

L. Huang, M. Hu, Z. Chen, H. Yu, and C. Liu, "Research on a direct-drive wave energy converter using an outer-pm linear tubular generator," IEEE Transactions on Magnetics, vol. 53, no. 6, pp. 1-4, 2017.

H. Polinder, M. E. Damen, and F. Gardner, "Linear PM generator system for wave energy conversion in the AWS," IEEE Transactions on Energy Conversion, vol. 19, no. 3, pp. 583-589, 2004. (26/04/2019). Ocean Power Technologies. Available:

https://www.oceanpowertechnologies.com/

Carnegie. (26/04/2019). Available:

https://www.carnegiece.com/wave/

J. K. Shek, D. E. Macpherson, M. A. Mueller, and J. Xiang, "Reaction force control of a linear electrical generator for direct drive wave energy conversion," IET renewable power generation, vol. 1, no. 1, pp. 17-24, 2007.

[10] X. Liu, C. Zou, Y. Du, and F. Xiao, "A linear consequent pole stator permanent magnet vernier machine," in Electrical Machines and Systems (ICEMS), 2014 17th International Conference on, 2014, pp. 1753-1756: IEEE.

[11] A. A. Almoraya, N. J. Baker, K. J. Smith, and M. A. H. Raihan, "Design and Analysis of a Flux Concentrated Linear Vernier Hybrid Machine with Consequent Poles," IEEE Transactions on Industry Applications, vol. In press, 2019.

[12] D. Li, R. Qu, J. Li, and W. Xu, "Consequent-pole toroidal-winding outer-rotor Vernier permanentmagnet machines," IEEE Transactions on Industry Applications, vol. 51, no. 6, pp. 4470-4481, 2015. Y. Gao, R. Qu, D. Li, J. Li, and G. Zhou, "Consequent-pole flux-reversal permanent-magnet machine for electric vehicle propulsion," IEEE

Transactions on Applied Superconductivity, vol. 26, no. 4, pp. 1-5, 2016. T. W. Ching, K. T. Chau, and W. Li, "Power factor improvement of a linear Vernier permanent-magnet 
machine using auxiliary DC field excitation," IEEE Trans. on Magn., vol. 52, no. 7, pp. 1-4, 2016.

[15] S. P. McDonald, N. J. Baker, M. Espinoza, and V. Pickert, "Power-Take-Off Topology Comparison for a Wave Energy Converter," presented at the IET Renewable Power Generation, Technical University of Denmark (DTU) 2018.

[16] T. Lettenmaier, A. Von Jouanne, and T. Brekken, "A new maximum power point tracking algorithm for ocean wave energy converters," International journal of marine energy, vol. 17, pp. 40-55, 2017.

[17] T. M. Lettenmaier, "Testing of wave energy converters using the Ocean Sentinel Instrumentation Buoy," Oregon State University, Oregon, US, 2013.

[18] B. Ding, B. S. Cazzolato, M. Arjomandi, P. Hardy, and B. Mills, "Sea-state based maximum power point tracking damping control of a fully submerged oscillating buoy," Ocean Engineering, vol. 126, pp. 299-312, 2016.

[19] X. Xiao, X. Huang, and Q. Kang, "A hill-climbingmethod-based maximum-power-point-tracking strategy for direct-drive wave energy converters," IEEE Transactions on Industrial Electronics, vol. 63, no. 1, pp. 257-267, 2015. 